Iván Jaksic y Juan Luis Ossa (editores), Historia política de Chile, 18102010. Tomo I. Prácticas políticas (Santiago: Fondo de Cultura Económica, 2017).

RESEÑA

\title{
LA HISTORIA POLÍTICA SE TOMA LA PALABRA
}

\author{
Manuel Vicuña \\ Universidad Diego Portales
}

D urante mucho tiempo, tal vez demasiado, la historia política despertó sospechas en varias provincias del mundo académico. No fueron tan raros quienes especularon con jubilarla. Para esto aducían el tradicionalismo de sus enfoques. Los juzgaban encandilados por el destino de los "grandes hombres" (papas, reyes, presidentes, ministros o parlamentarios), y presas de un protocolo de escritura que se conformaba con narrar acontecimientos con la parsimonia descriptiva de una crónica incapaz de calar el significado profundo de los fenómenos del pasado. Dicho en simple, la historia política fue desautorizada por otras corrientes historiográficas, como la historia de las mentalidades, la historia social, la historia cultural y un largo etcétera, que contempló fértiles cruces disciplinarios (por ejemplo, con la antropología simbólica) y asaltos teóricos procedentes de los cuarteles del postestructuralismo y sus aliados. Todas estas corrientes hicieron lo posible por irrigar en diversas direcciones, movidas por afanes imperialistas más o menos encubiertos, y por la convicción de representar un avance teórico y me-

Manuel Vicuña. Doctor en historia de la Universidad de Cambridge. Decano de la Facultad de Ciencias Sociales e Historia de la Universidad Diego Portales. Autor, entre otros libros, de La belle époque chilena. Alta sociedad y mujeres de elite en el cambio de siglo (2001, 2010); Un juez en los infiernos. Benjamín Vicuña Mackenna (2009), y Reconstitución de escena (2016).Email: manuel.vicuna@udp.cl. 
todológico con potencial para reformular el código epistemológico del conocimiento histórico.

La escuela francesa de los Annales, quizá la más influyente del siglo XX, a veces tendió a restarle protagonismo a la historia política, en la misma medida en que cuestionó el valor de los eventos, de la narrativa y del género biográfico. De esas reprobaciones queda poco o nada, precisamente porque quienes se interesaban en narrar acontecimientos y la vida de personajes mostraron cómo, haciendo algo en apariencia muy acotado, era posible dar cuenta de fenómenos más amplios, como el imaginario del mundo popular o el repertorio valórico de los señores del orden feudal. En el caso específico de la historia política, su retorno a la primera plana también respondió, entre otros motivos, a los desafíos planteados por las relaciones entre agencia y estructura y a la fascinación por la cultura política como universo simbólico al interior del cual los actores del pasado les otorgaban sentido a sus experiencias.

En países como Brasil, Argentina y Uruguay, y en menor medida en Chile, la renovación de la historia política, actualmente muy inclinada al diálogo con otras disciplinas y tendencias historiográficas, también ha sido abonada por el cultivo de la historia reciente, un campo de estudio al cual se han dirigido muchos de los académicos interesados en los avatares de la última oleada de dictaduras militares y de los procesos de transición a la democracia. Las subjetividades de los militantes, los traumas históricos derivados del terrorismo de Estado y las reelaboraciones de la memoria colectiva han concentrado la atención de investigadores ya habituados a conjugar las pesquisas documentales con la historia oral, y a perseguir los rastros de la política en espacios que antes se creían al margen de sus trayectorias.

En este contexto, el primer tomo de la Historia política de Chile, 1810-2010, ${ }^{1}$ centrado en las prácticas políticas, pretende contribuir a la renovación de la historia política referente a Chile, expandiendo su radio de acción "más allá de instituciones y organizaciones" (12). Se trata de un esfuerzo colaborativo serio, que responde a una rigurosa metodología de trabajo grupal, y promete instalarse como una obra de

${ }^{1}$ Iván Jaksic y Juan Luis Ossa (editores), Historia política de Chile, 18102010. Tomo I. Prácticas políticas (Santiago: Fondo de Cultura Económica, 2017). En adelante el libro se citará tan sólo con su número de página entre paréntesis. 
referencia. Dieciséis autores participaron en la redacción de los catorce capítulos de este volumen. Varios son especialistas en el tema tratado, de modo que los capítulos a su cuidado responden al balance de años de trabajo. Como suele ocurrir con los libros que reúnen colaboraciones de distintos autores, el nivel de los capítulos es dispar, aunque las fluctuaciones en calidad tienden a mantenerse en un reducido ancho de banda. Tomando distancia de los capítulos a fin de discernir los "problemas historiográficos" que los articulan con prescindencia de sus particularidades, el editor de este primer tomo, Juan Luis Ossa Santa Cruz, identifica cuatro cuestiones subyacentes: "revolución y guerra; política asociativa; participación social en la política nacional, y el binomio democracia y dictadura" (16).

\section{DIVERSIDAD DE EXPRESIONES POLÍTICAS}

La Historia política de Chile, 1810-2010 contempla una diversidad de perspectivas. Ésta permite ampliar el rango de las cuestiones tradicionalmente asociadas a la historia política, al mismo tiempo que expone los vínculos (a veces manifiestos, en otras ocasiones subterráneos) entre estas diferentes áreas de estudio. En atención a la particularidad de cada una de ellas, el libro no presenta una periodización uniforme. Cada autor elabora una división temporal propia, logrando un calce adecuado con la materia abordada. Esta opción metodológica permite perfilar procesos relevantes, evidenciar episodios críticos y trazar un cuadro de continuidades y rupturas.

Tratándose de una historia del Chile republicano, el volumen empieza con el estudio del proceso de Independencia — en realidad, una "guerra civil revolucionaria"-, de la cual se quiere hacer ver su carácter revolucionario, para después hacerse cargo de las cuatro guerras civiles (1829-30, 1851, 1859 y 1891) que acompañaron y trastornaron el proceso de consolidación del sistema institucional. La historiografía sobre la Independencia y las guerras externas presenta un mayor desarrollo que el estudio de los conflictos internos, por lo que otorgarles protagonismo resulta muy oportuno. Lo interesante del capítulo sobre este asunto, obra de Joaquín Fernández Abara, es que deja a un lado el énfasis en la dimensión militar de los conflictos, para centrarse en sus motivos y efectos políticos, en las relaciones entre oficialismo y oposi- 
ción, entre los poderes ejecutivo y legislativo, entre la clase dirigente y los notables locales y la expansión y la autonomización de la burocracia estatal, y entre centralismo y regionalismo. Todas las guerras civiles aluden a la precariedad del orden republicano, que se fracturó una y otra vez en respuesta a problemas a la vez constantes y variables. El poder del ejecutivo para manipular el resultado de las elecciones y el sesgo autoritario de sus actuaciones desataron crisis políticas que, junto con movilizar ejércitos, consolidaron un horizonte normativo proclive a la limitación del poder del gobierno en nombre de principios liberales.

Aquí se advierten dos afinidades electivas entre capítulos aparentemente inconexos. En "Democracia y dictadura en el Chile republicano. Prácticas, debates y conflictos políticos", Marcelo Casals rastrea, de modo convincente y conceptualmente sofisticado, el desarrollo de la "matriz liberal de la cultura política chilena" (353). Con su promesa de participación y actualización democrática, esa matriz se instituyó en consenso básico de todos los sectores políticos, obligando incluso a los regímenes autoritarios a rendirle honores, mañosamente, como ideal normativo. El estudio de las guerras civiles también puede asociarse con la investigación sobre la violencia política emprendida por Brian Loveman y Elizabeth Lira, un recordatorio de que la tan cacareada excepcionalidad del caso chileno en el contexto latinoamericano, excepcionalidad derivada de su temprana consolidación institucional y de la continuidad electoral de su tradición democrática, reposa en el uso de la violencia política en variadas versiones, y no sólo por parte del Estado o en nombre del orden público de acuerdo a los criterios del gobierno de turno.

Quizá podría pensarse buena parte del volumen desde esta perspectiva: las promesas, los avances sinuosos y los retrocesos abruptos en el proceso de actualización de la democracia, entendida como un horizonte normativo móvil, que reformula, con cada desplazamiento, los alcances del régimen republicano adoptado a propósito de la Independencia. Varios capítulos aluden, directa o indirectamente, a este fenómeno. El texto de Andrés Baeza Ruz, por ejemplo, explora las asociaciones que, por fuera o en comunicación con el sistema de partidos, van dándole densidad a la sociedad civil. En ese espacio intermedio entre el Estado y el mercado se desenvuelven procesos de constitución de ciudadanía más allá del circuito electoral, más allá del juego político en sentido es- 
tricto, en tanto aglutinan a individuos de otro modo impotentes en torno a causas comunes, y de paso ofrecen herramientas para interpelar a las autoridades y forjar identidades colectivas.

En sintonía con este capítulo, el trabajo de Eduardo Posada Carbó ahonda en las vías de maduración de prácticas ciudadanas, cuyo radio de influencia alcanzó desde temprano a sectores privados del derecho a voto, en el marco de un calendario electoral intenso, a veces incluso congestionado, que se mantiene vigente, salvo por algunas coyunturas críticas, entre 1833 y 1970. Inspirado en el trabajo de Frank O'Gorman, Posada Carbó sabe que el estudio de la participación electoral no puede restringirse al análisis del número de votantes efectivos, porque las formas de movilización asociadas a esas contiendas imantan, y por lo tanto politizan, a sectores en principio al margen de la política partidista, como fue el caso, durante más de un siglo, de las mujeres y de los analfabetos. A ratos, Posada Carbó enlaza con los intentos por dar cuenta de la "cultura de la movilización política", aunque sin aludir a uno de los grandes motores detrás de ella: la oratoria como una práctica destinada a persuadir al intelecto y movilizar los afectos, al extremo de constituir comunidades basadas en ideas y sentimientos compartidos, con repercusiones en el repertorio de la acción colectiva.

Bajo el prisma de la actualización de la democracia como clave de acceso al interior del libro, también hay que destacar el trabajo de María Rosaria Stabili sobre la "res-pública de las mujeres". Stabili aborda las formas de movilización, los actos de toma de palabra, los tipos de recursos asociativos y los recursos argumentales utilizados en la lucha por la conquista de derechos (políticos, civiles y sociales), cruzando todas estas variables con los clivajes del sistema de partidos, el tejido y las desgarraduras del mundo asociativo, y la tensión entre demandas de género, con liderazgos femeninos autónomos, y programas de acción supeditados a otras agendas, como las reivindicaciones de clase, históricamente más funcionales a los intereses masculinos. De nuevo, se trata de mapear "escuelas de ciudadanía" y fuentes de agencia orientadas a extender derechos, consumar promesas y enriquecer la representación y la participación política como instancias de ejercicio democrático. En el fondo, este volumen se propone ampliar el elenco de la historia política, o, para ser más precisos, reconocer la coexistencia y conexión de escenarios diversos, que comprometen las actuaciones de las élites tra- 
dicionales, de las clases medias, de los sectores obreros, de las mujeres y del campesinado, en una obra de estructura coral, con narrativas que a ratos se entrelazan y se anudan, a medida que avanza la lectura de los capítulos.

Esa heterogeneidad social y política se expresa de distintos modos. Una de las vertientes más interesantes de la misma alude a la condición plural de la clase media. El estudio de las clases medias realizado por Elizabeth Quay Hutchison y María Soledad Zárate Campos es uno de los puntos altos del libro. En pleno dominio de la bibliografía pertinente sobre América Latina y Chile, este capítulo pone el acento en el periodo que va de los años veinte a los setenta del siglo XX, logrando identificar las fuerzas motrices del crecimiento y de la diversificación de las clases medias, a la vez que enriquece el análisis introduciendo la variable de género, iluminando por esta vía una dimensión de las relaciones de poder comúnmente descartada por la bibliografía sobre el tema. Hutchison y Zárate combinan el análisis de la estructura social y de los aspectos relacionales en la conformación de identidades colectivas, con la sociología de las profesiones y el estudio de los repertorios valóricos, de los niveles de ingreso y de la afiliación a partidos situados a todo lo ancho del espectro político. En otras palabras, atacan el tema desde distintos frentes, y concertadamente, intersectando la trayectoria de las clases medias con el desarrollo de la burocracia estatal y, sobre todo, con la historia del Estado de bienestar y del proyecto modernizador que lo inspiró.

La nueva historia política, vuelvo a señalarlo, busca mostrar cómo la política también se desenvuelve en ámbitos poco transitados por la historiografía hasta hace unas décadas. La cultura es una de esas áreas antes periféricas, que la expansión del estudio de la política ha ido incorporando al centro de sus preocupaciones. ¿Cómo crear comunidades nacionales que tributen a una coalición o a una clase social, en su afán por hegemonizar el espacio público e imprimirle su sello al orden institucional? En esta línea, el trabajo de Patrick Barr-Melej, "Manipulando el 'alma del pueblo': Cultura y prácticas políticas en Chile contemporáneo", explora la dimensión política de la cultura a través del análisis del folclor como capital simbólico, usado para validar proyectos políticos con toques de nacionalismo, que alegaban representar, en beneficio propio, lo auténticamente chileno. El repertorio de las 
tradiciones y los personajes del campo, la llamada "cultura huasa" originalmente idealizada por el criollismo, durante el siglo XX se constituyó en parte importante del menú de las masas urbanas. De ahí el interés, común a las fuerzas de izquierda, de centro y de derecha, por apropiarse de esa fuente de legitimidad social redituable en términos políticos y éticos.

El folclorismo remite al campo desde una perspectiva urbana, pero el campo, en este tomo, no es sólo el patrimonio disputado en el marco de las "políticas de la cultura" activadas para impulsar o contener las transformaciones de signo reformista o revolucionario. En el último capítulo del libro, Claudio Robles Ortiz se ocupa de la sociedad rural, y de sus relaciones de ida y vuelta con la política nacional, desde la segunda mitad del siglo XIX hasta la reforma agraria, sin pasar por alto la micropolítica acotada a espacios locales de dominación, contestación y negociación. Hacendados, medieros, inquilinos, peones afuerinos, militantes de partidos, agentes de sindicatos y funcionarios públicos conforman la nómina de actores que giran en torno a las vicisitudes del sistema de la hacienda; a la conformación de un "movimiento campesino", menos tardío de lo esperado; al destino accidentado de la sindicalización campesina, trabada por acuerdos políticos autorizados en nombre de los requerimientos del proyecto desarrollista, y a las tácticas de resistencia al poder patronal (disimuladas al principio, desembozadas después) de los obreros agrícolas y los inquilinos cuyas condiciones laborales fueron deteriorándose con el avance del capitalismo agrario en el valle central.

\section{PROMESAS INCUMPLIDAS}

"Las prácticas políticas", observa Robles Ortiz, "pueden ser definidas como las formas de acción colectiva por medio de las cuales distintos actores participan en las instancias y ámbitos en los que tienen lugar el ejercicio, la negociación y la contestación del poder. Dada la complejidad que puede alcanzar el repertorio de formas de acción colectiva, la agencia de los actores involucrados en esas dimensiones de la acción política construye una 'arena de poder' compleja, formada por una multiplicidad de escenarios concretos, desde el nivel nacional de la política en las instituciones centrales del Estado hasta los espacios polí- 
ticos locales" (423). La definición de prácticas políticas, considerando el subtítulo del volumen, debiese haber ocupado un lugar central en la trama teórica que sostiene los distintos capítulos. Cuesta entender por qué hay que llegar al último texto del libro para encontrar la formulación más clara del asunto. Los libros académicos deben ser evaluados, como mínimo, teniendo en cuenta esta pregunta: el texto, ¿de verdad entrega todo lo que compromete?

Esta historia política de Chile, aunque meritoria en muchos aspectos, defrauda algunas de sus mayores promesas. La escasa elaboración del concepto de prácticas políticas obliga a preguntarse, en el curso de varios capítulos, exactamente en qué pueden haber consistido; y ese vacío, en los pasajes más flojos del volumen, que son los destinados al análisis de los partidos políticos en Chile, nos deja librados a la lectura de un tipo de historia cuyo convencionalismo contradice las pretensiones renovadoras de este trabajo colectivo. Si las prácticas políticas reclaman el centro de la atención, ¿tiene sentido explorar la penetración capilar de los partidos en la sociedad chilena, sin ocuparse, por ejemplo, del alcance de las redes del clientelismo?

Otra promesa defraudada, de modo todavía más flagrante: la convicción de estar cultivando un tipo de historiografía enriquecida con los aportes de los estudios comparados y transnacionales. En el último tiempo, el calificativo "transnacional" se ha convertido en una vistosa etiqueta, a menudo adoptada de modo irreflexivo, en el empeño por revestirse del aura que, desde la década de 1990, otorga el producto de moda en el mercado académico. El pecado que atormenta a los historiadores de hoy, descolocados por el "giro transnacional": confinar sus narrativas a la historia del Estado-nación, como si esta formación histórica, bastante reciente, fuese la unidad básica de análisis o el contenedor natural en cuyo interior reside el secreto para la comprensión de una multiplicidad de fenómenos tan relevantes en el pasado como en el presente. Quienes practican la perspectiva transnacional (muy interesados en cuestiones como las diásporas y la circulación de ideas y objetos a través de fronteras varias) han resultado persuasivos a la hora de exponer las limitaciones de un enfoque historiográfico que creció de la mano del nacionalismo, o por lo menos a su sombra. La heterodoxia transnacional de los años noventa va camino de situarse en el lugar de la ortodoxia, si es que ya no lo ha hecho. En este panorama, los historiadores chilenos han destacado por 
su fidelidad al modelo de una historia nacional introvertida, poco dada a levantar la vista para establecer comparaciones que ayuden a distinguir lo singular de lo compartido con otras experiencias.

Los historiadores congregados en este volumen desaprovecharon (salvo por algunos momentos fugaces, destellos que se extinguen en segundos de lectura) la oportunidad de remediar esa insuficiencia. Los editores del proyecto, impulsados por la necesidad de dilucidar el problema del "excepcionalismo chileno", solicitaron a los autores incorporar "elementos comparativos y transnacionales" a sus trabajos. Hay que decir que los autores no respondieron a la solicitud, y, por lo visto, los editores optaron por hacerse los desentendidos. Abundan los ejemplos para acreditar esta afirmación; comienzo con uno, a mi juicio muy ilustrativo, porque alude al propio editor del presente volumen. En el primer capítulo del libro, Ossa Santa Cruz reduce su vocación comparatista a esta expresión: "La revolución en Chile tuvo muchas similitudes, pero también muchas diferencias, con otras zonas de Sudamérica" (30). ¿Cuáles son esas similitudes? ¿Cuáles son esas diferencias? Silencio.

Existen varias encrucijadas análogas a lo largo del libro, pasajes en los que el mismo despliegue de los argumentos invitaba, casi sin necesidad de insinuarse, a cederles la palabra a las aproximaciones comparatistas o transnacionales. ¿Cómo no hacerlo al hablar del papel de la prensa como "actor clave en la formación de la nueva comunidad política" (212), teniendo en cuenta que el primer periódico chileno nació en 1812, mientras en las capitales virreinales la prensa llevaba décadas y décadas de ventaja en la carrera por la articulación de una esfera pública ilustrada? ¿Cómo no adentrarse en las implicancias del internacionalismo obrero, de manifiesto en la presencia en Chile de la organización anarcosindicalista International Workers of the World, y en la bolchevización del Partido Obrero Socialista? Al ocuparse de la reforma agraria en Chile, ¿no cae de cajón insertar ese proceso en un marco explicativo amplio y diverso, reparando en otras experiencias análogas, y no sólo en América Latina, además de remitir el análisis a las dinámicas de la Guerra Fría y a los esfuerzos de contención revolucionaria por parte de Estados Unidos, esfuerzos concretados en iniciativas como la Alianza para el Progreso, que apostó a la "Revolución en libertad" de la DC como camino alternativo al modelo de la Revolución Cubana? Si la pre- 
tensión era contrarrestar el ensimismamiento de la historiografía nacional, ¿basta con describir determinadas coyunturas y fenómenos como ecos locales de fenómenos globales?

Tampoco pueden desconocerse las omisiones bibliográficas y temáticas. Hay libros que atacan la médula de los tópicos elegidos en este volumen, cuya ausencia acaba por debilitar su contextura. Aclaro que no hablo, necesariamente, de literatura publicada antes de ayer; hablo de "clásicos", incluso, con décadas de historia. Basta pensar en Political Brokers in Chile: Local Government in a Centralized Polity (1977), del politólogo Arturo Valenzuela, una referencia obligada en el estudio del rol del clientelismo como eslabón de la cadena de representación política que ataba el destino de la política local a la política nacional. Igual de injustificable parece la exclusión de Timothy R. Scully, con su libro Rethinking the Center: Party Politics in Nineteenth-and Twentieth-Century Chile (1992), debido a su destreza para analizar el desarrollo del sistema de partidos con una lógica dinámico-estructural que revela su carácter tripartito, los clivajes que movilizan a sus actores y las funciones del centro político como eje articulador y, más tarde, factor de polarización de la democracia chilena. Otra ausencia curiosa: Carmen Mc Evoy, con su texto Guerreros civilizadores. Política, sociedad y cultura durante la Guerra del Pacifico (2011), en cuyas páginas se aborda, con lujo de detalles, tanto la explosión de asociatividad cívica detonada por la guerra - base de un despliegue de prácticas políticas que activaron formas inusitadas de patriotismo local-, como el protagonismo de la prensa en la movilización de recursos, en la legitimación del conflicto arguyendo su carácter de cruzada civilizatoria, y en el consiguiente compromiso emocional de la ciudadanía, en una encrucijada crítica de la historia de Chile, con repercusiones de largo plazo en la fisonomía adquirida por el Estado, en el carácter de la economía chilena y en la consolidación de las clases medias.

Ahora observaremos las omisiones temáticas. Sólo señalaré algunas que apuntan al núcleo expositivo de los capítulos, no a su periferia, porque en estos casos las omisiones resultan comprensibles atendidas las limitaciones de espacio. Parto con el capítulo sobre la prensa, a cargo de Carla Rivera, de lo mejor del libro. En él se echa de menos el tratamiento de la prensa obrera, vital en la diversificación de la esfera 
pública, en la circulación de nuevos referentes ideológicos como pilares de un perdurable discurso contra-hegemónico, y en la constitución de un sujeto político capaz de acomodar en su interior buena parte de la heterogeneidad sociológica del mundo popular. Líderes como Luis Emilio Recabarren, tipógrafo de oficio, y un periódico como El Despertar de los Trabajadores, caballo de batalla de su programa emancipador, no tienen cabida en esta narrativa. Tampoco queda claro por qué se dejó a un lado el análisis de la "Lira popular", artífice y representante de una sensibilidad plebeya crítica, que usó las décimas de origen medieval, vendidas por miles en pliegos rústicos, como medio de irrisión de los sectores dirigentes.

Otra omisión, emparentada temáticamente con las anteriores, se advierte en el texto sobre el asociacionismo político, de Andrés Baeza Ruz, también muy logrado, pese a todo. Como era esperable, el autor destina una atención preferente a la historia del mutualismo y del movimiento obrero, pero no se refiere al conjunto de prácticas implementadas por parte de sus organizaciones, para promover la constitución de ciudadanos maduros y, por lo tanto, con mayor capacidad de interlocución con la clase dirigente y arrastre en la base social. Pienso en las iniciativas destinadas a la "redención moral" del pueblo según parámetros ilustrados, algo que supuso esfuerzos por modificar las formas de sociabilidad popular, promover el uso autónomo del voto y propagar la alfabetización como antesala de la concientización de clase. Todo esto derivó en el ejercicio de prácticas políticas a nivel micro (controlar el consumo de alcohol y la afición a los juegos de azar, forzar el envío de los hijos a la escuela y formar liderazgos con capacidades oratorias), las que tenían como finalidad dotar de mayor agencia histórica a las luchas reivindicativas asociadas a la "cuestión social". Todavía menos entendible resulta el descarte del programa de "Promoción popular" llevado adelante por el gobierno de Eduardo Frei Montalva, que significó la propagación de organizaciones de base (léase, juntas de vecinos y centros de madres), cuyo objetivo era la incorporación política y la cooptación electoral de sectores hasta entonces relegados a los márgenes de la actividad democrática.

En todo caso, ninguna de las objeciones anteriores debiese desincentivar la lectura del primer tomo de la Historia política de Chile, 1810-2010. Prácticas políticas. Los aportes superan por mucho a las 
insuficiencias. Los temas tratados ofrecen un espectro amplio, que le otorga a la política una condición multidimensional imprescindible si queremos hacerle justicia y comprenderla. Quien lea este volumen de principio a fin seguramente quedará a la espera de los restantes. EP 